\title{
Qualidade nutricional de frutos da pupunheira vermelha integral desidratados a diferentes temperaturas ${ }^{1}$
}

\author{
Reginaldo Ferreira da Silva², Marilia Temporim Furtado², Doralice Pereira Rodrigues ${ }^{2}$
}

Resumo: Após a desidratação do alimento, se faz necessário uma avaliação nutricional, já que, altas temperaturas e tempos longos de secagem induzem a perdas de nutrientes. Sendo assim, o objetivo deste trabalho foi determinar em qual temperatura de secagem a polpa da pupunha vermelha integral, sofre menores perdas no seu conteúdo nutricional. As temperaturas de secagem utilizada foram, 45,55 e $65^{\circ} \mathrm{C}$. A temperatura mais eficiente na retirada de água livre da polpa de pupunha foi a $65^{\circ} \mathrm{C}$. Apesar dos lipídeos apresentarem-se mais concentrados a temperatura de $45^{\circ} \mathrm{C}$, foi a $65^{\circ} \mathrm{C}$ que houve maior concentração de proteínas e fibras. A polpa desidratada a 55 e $65^{\circ} \mathrm{C}$, podem ser consideradas como fonte de fibra alimentar segundo a legislação. O menor teor de cinzas, foi obtido no tratamento a temperatura de $45^{\circ} \mathrm{C}$. Os carboidratos e os carotenóides foram significativamente influenciados pelas maiores temperaturas de secagem. Portanto, dependendo da finalidade nutricional ou tecnológica para desidratar o fruto de pupunha, as três temperaturas analisadas nessa pesquisa, devem ser utilizadas para atingir o objetivo proposto.

Palavras-chave: Desidratação de alimentos; Farinha vegetal; Composição centesimal.

\section{Nutritional quality of fruits of whole red peach palm dehydrated at different temperatures}

\begin{abstract}
After dehydrating the food, a nutritional assessment is necessary, since high temperatures and long drying times induce nutrient losses. Therefore, the objective of this work was to determine at which drying temperature the pulp of the whole red peach palm suffers less losses in its nutritional content. The drying temperatures used were 45,55 and $65^{\circ} \mathrm{C}$. The most efficient temperature in removing free water from the peach palm pulp was $65^{\circ} \mathrm{C}$. Despite the lipids proved more concentrated at $45^{\circ} \mathrm{C}$, it was at $65^{\circ} \mathrm{C}$ that there was increased concentration of both proteins and fibers. The pulp dehydrates at 55 and $65^{\circ} \mathrm{C}$, they can be considered as a source of dietary fiber according to the legislation. The lowest ash content was obtained in the treatment at $45^{\circ} \mathrm{C}$. Both the carbohydrates and carotenoids were significantly influenced by the highest drying temperatures. Therefore, depending on the nutritional or technological purpose to dehydrate the peach palm fruit, the three temperatures studied in this work, must be used to achieve the proposed objective.
\end{abstract}

Keywords: Food dehydration; Plant flour; Proximate composition.

${ }^{1}$ Submetido em 13/08/2020 e aprovado em 16/12/2020;

${ }^{2}$ Universidade Federal do Acre, Centro de Ciências da Saúde e do Desporto, Rio Branco, Acre, Brasil; E-mail: reginaldo.alimentos@ gmail.com (Autor correspondente) - ORCID: https://orcid.org/0000-0002-7010-6279; matemporim@ @mail.com - ORCID: https://orcid.org/0000-00031268-7359; doralicepr@outlook.com - ORCID: https://orcid.org/0000-0001-6618-4701

Agropecuária Técnica, Areia-PB, v. 41, n. 3-4, p. 101-108, 2020

https://doi.org/10.25066/agrotec.v41i3-4.54435 


\section{Introdução}

A pupunheira (Bactris gasipaes Kunth), nativa da região amazônica é uma palmácea apreciada na alimentação pelo seu palmito e pelos seus frutos, com potencialidade para a produção sustentável de maneira econômica e ambiental. O fruto pode ser consumido após cozimento ou desidratados e utilizados como ingrediente para elaboração de diversos produtos alimentícios, tais como: panetones (Oliveira te al., 2010); mix de farinha de trigo e polpa de pupunha (Medeiros, et al., 2012); bolos (Kaefer, et al., 2013); biscoitos (Reck, et al., 2016) e pães (Lima et al., 2020). Entretanto, para ter matéria-prima o ano todo, entre uma safra e outra, é necessário que a polpa do fruto, seja conservada para tais finalidades alimentares.

Os maiores plantios dessa palmeira estão nos países do norte da América do Sul e nos Países da América Central (Oliveira e Marinho, 2010; Santos et al., 2017). A importância dessa palmeira cresceu consideravelmente no Brasil, principalmente para produção de frutos e palmito, sendo cultivada na Amazônia comumente por agricultores familiares (Carvalho et al., 2013).

O fruto da pupunha possui boa qualidade nutritiva e energética, principalmente, em relação a vitamina $\mathrm{A}$, cálcio, fósforo, ferro, complexo $\mathrm{B}$, carboidratos e gorduras insaturadas (Girón et al., 2017). Corroborando com esta afirmativa, RojasGarbanzo et al. (2016) também destacaram o potencial do fruto em termos de propriedades antioxidantes. Entretanto, o fruto só deve ser consumido após ser submetido ao processo de cocção "cozido ou desidratado", em função da presença de compostos fenólicos que são inativados, atenuando o sabor adstringente presente na casca e polpa.

Atualmente, a produção de farinhas obtidas da desidratação de frutos "polpa ou integral, polpa e casca" proporciona grande diversidade de matéria-prima para a indústria de alimentos, panificáveis, produtos dietéticos, dentre outros, por apresentarem alto teor de amido, fibras dietéticas e sais minerais. Sendo assim, a farinha de pupunha desidratada poderá servir como matéria prima a ser utilizada em determinadas épocas do ano como principal ingrediente na fortificação de alimentos panificáveis e outros. Segundo Oliveira e Marinho (2010), diversos tipos de farinhas obtidas de frutos regionais ou mesmo farinhas integrais vem sendo utilizadas com essa finalidade.

O processo de secagem é uma técnica antiga utilizada como forma de desidratar os alimentos com objetivos de facilitar a conservação do produto; estabilizar os componentes aromáticos à temperatura ambiente por longos períodos de tempo; proteger contra degradação enzimática e oxidatíva; reduzir o seu peso; economizar energia por não necessitar de refrigeração e poder disponibilizar o produto durante qualquer época do ano (Park et al., 2001).

Levando em consideração as propriedades nutricionais e funcionais do fruto de pupunha já citados na literatura, a disponibilidade do fruto na região e todas as vantagens atribuídas ao processo de desidratação de alimentos, Sendo assim, o objetivo com este trabalho foi determinar em qual temperatura de secagem a polpa da pupunha vermelha integral, sofre menores perdas no seu conteúdo nutricional.

\section{Material e Métodos}

\subsection{Aquisição e processamento dos frutos}

Foram adquiridos $40 \mathrm{~kg}$ de pupunha no Mercado Municipal Elias Mansur em Rio Branco - AC. Os frutos apresentavam estádio de maturação comercial, caracterizado pela cor vermelha da casca, com tamanhos e formatos uniformes. Os cachos de pupunha foram transportados imediatamente ao laboratório de Tecnologia de Alimentos - UTAL, da Universidade Federal do Acre, onde foi realizado o experimento. Os frutos foram lavados em água corrente, higienizados com água clorada a 150 ppm por 20 minutos. Em seguida, foi coletado uma amostra de frutos para realizar análises da composição físico-química a temperatura ambiente local $28^{\circ} \mathrm{C}$, marcado em aparelho termo higrômetro digital com sensor externo AK28 new. O restante dos frutos foram aquecidos por 20 minutos em água fervente e resfriados em água gelada. Depois, foram cortados manualmente em bandas, com casca, e retirado as sementes com faca de aço inox, pesados, distribuídos em bandejas e submetidos à secagem. Para cada tratamento, foram utilizados 2 quilos de frutos em 3 bandejas, sendo seis quilos por tratamentos, considerando o intervalo de temperatura de desidratação de $10^{\circ} \mathrm{C}$ entre si, até peso constante, no seguinte esquema fatorial $(3 \times 3)$, com três 
temperaturas, 45,55 e $65^{\circ} \mathrm{C}$ e três repetições de 2 quilos de frutos por bandeja, desidratados em estufa com circulação de ar forçada. No final da secagem, os 6 quilos de polpa de pupunha in natura de cada tratamento, resultaram em médias de 500, 540 e 400g de polpa de pupunha integral desidratada, respectivamente. Para melhor entendimento do processo de obtenção da farinha de pupunha desidratada integral, o fluxograma descrito na figura 1 , mostra todas as etapas.

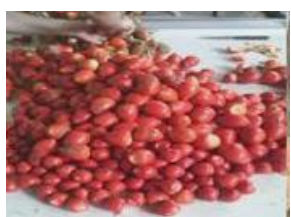

Pupunha in natura com casca

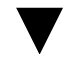

Pré-lavagem e pesagem

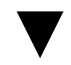

Sanitização: (água clorada / 20 minutos)

Cocção: (Água fervente / 20 minutos)

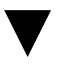

Resfriamento / 12 horas

$\checkmark$

Descasque e retirada de sementes

$\checkmark$

Secagem a diferentes temperaturas

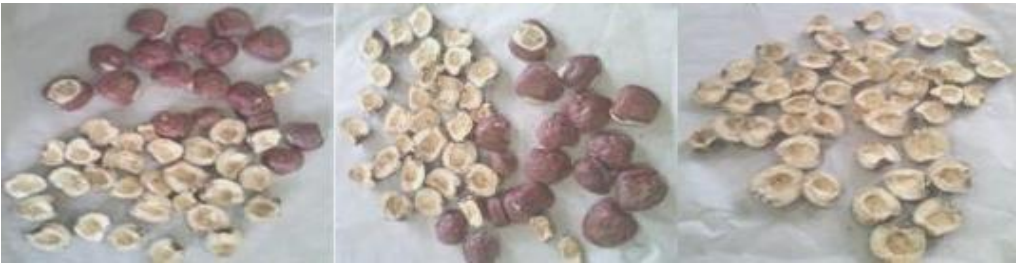

$45^{\circ} \mathrm{C}$

$55^{\circ} \mathrm{C}$

$65^{\circ} \mathrm{C}$

Armazenamento a $\pm 10^{\circ} \mathrm{C}$ por 2 dias até início das análises

Análises de composição físico-químicas e química

Figura 1 Fluxograma do processamento de frutos da pupunha vermelha para obtenção da farinha desidratada integral.

\subsection{Análises físico-químicas e estatística}

As análises da polpa de pupunha in natura e desidratadas foram realizadas no Laboratório de Físico-Química da Unidade de Tecnologia de Alimentos (UTAL) da UFAC. As seguintes determinações foram realizadas em triplicata segundo método proposto pelo Instituto Adolfo
Lutz - IAL (2008): Teor de umidade foi obtido por dessecação do material em estufa com circulação de ar, a $105^{\circ} \mathrm{C}$ até peso constante; o teor de lipídio foi determinado de acordo com o método de extração em aparelho Soxhlet, o resíduo extraído foi levado à estufa a $105^{\circ} \mathrm{C}$ por cerca 24 horas, resfriado em dessecador por 
aproximadamente 30 minutos e pesado; o teor de proteína foi determinado a partir da matéria seca, onde a amostra sofreu processos de digestão, destilação e titulação, de acordo com o método de Kjeldahl; a determinação de cinzas foi realizada em mufla a $550^{\circ} \mathrm{C}$ por 24 horas, em seguida foi resfriada em dessecador até temperatura ambiente e pesada; as fibras brutas foram obtidas pelo método de Weende, na qual se dissolve a amostra em solução ácida, e o que não é degradado constitui as fibras totais; os carboidratos foram obtidos por diferença a partir da seguinte fórmula: (\%) carboidratos $=100-$ (umidade + lipídios + proteínas + fibras + cinzas). As análises de carotenóides totais foi realizado de acordo com a metodologia descrita por Rodrigues-Amaya (2004) e os resultados foram expressos em $\mu \mathrm{g} / \mathrm{g}$ de beta caroteno e seus teores foram calculados segundo a fórmula $\mu \mathrm{g} / \mathrm{g}=(\mathrm{A} \times \mathrm{V} \times 1.000 .000)$ : $\mathrm{A}^{1 \% 1 \mathrm{~cm}} \times \mathrm{XMx} 100$.

Para a obtenção de dados da composição físico - químicas e química aproximados, foram feitas análises de variância (ANOVA), com posterior comparação das diferenças entre as médias pelo teste de Scott-Knott ao nível de 5\% de probabilidade, utilizando o programa estatístico SISVAR Ferreira, (2000).

\section{Resultados e Discussão}

O resultado da composição físico-química da polpa de pupunha vermelha in natura $\mathrm{e}$ desidratada integral a diferentes temperaturas de secagem, estão descritas na Tabela 1 .

Tabela 1 Composição físico-química da polpa de pupunha vermelha in natura e desidratada integral à diferentes temperaturas de secagem

\begin{tabular}{lcccc}
\hline & \multicolumn{4}{c}{ Temperaturas $\left({ }^{\circ} \mathrm{C}\right)$} \\
\hline Composição físico-química (\%) & In natura & \multicolumn{3}{c}{ Desidratação } \\
\hline Umidade & 28 & 45 & 55 & 65 \\
Proteína bruta & 61,8 & $11,16 \mathrm{a}$ & $10,50 \mathrm{~b}$ & $9,85 \mathrm{c}$ \\
Fibra alimentar & 2,6 & $5,42 \mathrm{~b}$ & $5,90 \mathrm{~b}$ & $6,80 \mathrm{a}$ \\
Cinzas & 1,9 & $2,60 \mathrm{c}$ & $3,0 \mathrm{~b}$ & $3,70 \mathrm{a}$ \\
Lipídeos & 0,7 & $1,63 \mathrm{~b}$ & $1,86 \mathrm{a}$ & $1,89 \mathrm{a}$ \\
Carboidratos & 2,2 & $12,03 \mathrm{a}$ & $11,75 \mathrm{a}$ & $11,81 \mathrm{a}$ \\
Carotenóides totais $\mu \mathrm{g} \cdot \mathrm{g}^{-1}$ & 30,8 & $67,16 \mathrm{a}$ & $66,99 \mathrm{a}$ & $65,95 \mathrm{~b}$ \\
\hline
\end{tabular}

Médias seguidas pela mesma letra, na linha, não diferem entre si pelo teste de Scott-Knott $(\mathrm{p} \leq 0,05)$.

\subsection{Teor de umidade}

Os teores de umidade da pupunha desidratada, diminuíram a medida que a temperatura foi aumentada, resultando em médias de 11,16, 10,50 e 9,85\%, respectivamente (Tabela 1). A diminuição nos teores de umidade, pode estar relacionada com o aumento da pressão de vapor parcial interna proporcionada pelo aumento de temperatura que aumenta a diferença entre a pressão de vapor do ar de secagem e do produto e assim, remove a água com mais facilidade e rapidez (Kumar et al., 2014). Os resultados obtidos no presente estudo, estão acima do intervalo de umidade de 7,2 a 9,3\% para pupunha e pequi, desidratados a $60^{\circ} \mathrm{C}$ (Goia et al.; 1993; Gallardo e Sierra, 1993; Parente et al., 2017). Os resultados citados anteriormente, ficaram abaixo dos obtidos no presente estudo. Entretanto, o teor de umidade da polpa desidratada em relação as temperaturas utilizadas, estão abaixo do limite máximo de $15 \mathrm{~g} 100 \mathrm{~g}^{-1}$, recomendado pela RDC $\mathrm{n}^{\circ} 263$ (Brasil, 2005).

\subsection{Proteínas totais}

A secagem aplicada a alimentos, além de concentrar as proteínas totais, também pode modificar sua estrutura química. As proteínas da pupunha desidratada, apresentaram teores de $5,42,5,90$ e $6,80 \%$, com o aumento da temperatura de secagem, 45, 55 e $65^{\circ} \mathrm{C}$, respectivamente (Tabela 1). As polpas desidratadas a 45 e $55^{\circ} \mathrm{C}$, não apresentaram diferenças estatísticas entre si. Entretanto, a $65^{\circ} \mathrm{C}$, a concentração foi maior em relação aos outros dois tratamentos, o que casou diferença significativa. Isso pode ser justificado, pela maior eficiência da retirada de água livre da polpa e consequentemente maior concentração da proteína bruta. A literatura cita valores de proteínas para pupunha desidratada sem casca e com casca, variando na faixa de 4,1 a 6,6\% (Goia 
et al., 1993; Kaefer et al., 2013), resultados próximos aos encontrados no presente estudo. Souza et al. (2019), desidratando frutos de pequi a diferentes temperaturas de secagem, 40, 50, 60 e $70^{\circ} \mathrm{C}$, cita que a temperatura de $60^{\circ} \mathrm{C}$, não afetou a concentração de proteínas do mesocarpo do fruto, mantendo assim, sua qualidade nutricional.

\subsection{Fibra dietética total}

Os percentuais de fibras alimentar, presente nos frutos de pupunha desidratada integral, apresentaram valores médios variando entre 2,60, 3,0 e $3,70 \%$, respectivamente, diferindo estatisticamente entre si. Esses teores estão dentro da faixa de variação citadas para frutos de pupunha processada com casca e sem casca, 0,8 a 9,3\% (Goia et al., 1993; Ferreira e Pena, 2003; Kaefer et al., 2013). Sabe-se, que os teores de fibra alimentar presente nos frutos, são influenciados principalmente pela espécie vegetal, tipos de fibras e composição das frações solúveis e insolúveis, processamento dos frutos com casca ou sem casca, dentre outros. Segundo Brasil (1998), alimento sólido que contém 3g/100 $\mathrm{g}$ de amostra é considerado como fonte de fibra alimentar. Sendo assim, apenas os frutos de pupunha integral que foram desidratados a temperatura de $45^{\circ} \mathrm{C}$, está abaixo do teor mínimo de fibras que regulamenta a legislação.

\subsection{Cinzas totais}

As polpas da pupunha desidratada, apresentaram os seguintes teores de cinzas, 1,63, 1,86 e $1,89 \%$, respectivamente, diferindo significativamente, apenas para a temperatura de $45^{\circ} \mathrm{C}$ em relação as outras duas (Tabela 1). Esses valores, estão próximos da faixa de intervalo citados na literatura, variando entre 1,09 a 2,20\% (Andrade et al., 2003; Oliveira et al., 2007; Carvalho et al., 2009; Silva et al., 2013; Kaefer et al., 2013). Porém, a cinza total obtida pela incineração dos alimentos, não tem necessariamente a mesma composição que a matéria mineral original, presente nos mesmos, pois pode haver perdas por volatilização ou algumas outras interações entre os diversos constituintes químicos presente na amostra (Cecchi, 2003). A legislação RDC $\mathrm{n}^{\circ}$ 263, ANVISA, (2005), determina teores de cinza em vegetais, até $4 \%$. Sendo assim, os teores de cinzas presente nas polpas de pupunha desidratadas do presente estudo, estão abaixo do limite máximo permitido na legislação.

\subsection{Lipídeos totais}

Os teores de lipídeos totais, para as três temperaturas, não diferiram estatisticamente entre si, apesar de se observar um maior percentual, $(12,03 \%)$ de lipídeos na pupunha desidratada a $45^{\circ} \mathrm{C}$, com a menor temperatura de secagem em relação aos outros dois tratamentos. Possivelmente, a temperatura mais baixa apresentou a menor influência no grau de saturação dos lipídeos e consequentemente de oxidação, o que proporcionou essa pequena diferença. Estudos mostram que existem diferenças significativas nos teores de lipídeos em frutos de pupunha desidratados com casca e sem casca, variando de 5,74 a 11,56\% (Oliveira et al., 2007; Carvalho et al., 2009; Kaefer et al., 2013). Os resultados obtidos para teores de lipídeos nesse estudo, estão próximos do maior teor de lipídeo descrito pelos autores citados.

\subsection{Carboidratos totais}

Os teores máximos de carboidratos, 67,91 e $66,99 \%$ foram obtidos a temperaturas de 45 e $55^{\circ} \mathrm{C}$ e, a $60^{\circ} \mathrm{C}$, tenderam a diminuir, o que causou diferença significativa em relação aos outros dois tratamentos. Os carboidratos presentes nos frutos podem ser influenciados pela temperatura de secagem, já que os mesmos participam de reações de escurecimento não enzimáticos como a reação de "Maillard e Caramelização". Entretanto, esses valores, estão dentro da faixa ou próximos aos descritos por (Clemente, 1991, 59,7 a $81 \%$ e Ferreira e Pena, 2003, 64,7\%) e, estão um pouco abaixo dos citados por Kaefer et al., (2013) para farinha de pupunha com casca, $(71,08 \%)$ e sem casca, $(73,13 \%)$. O tipo de açúcar presente no fruto, também determina o grau de influência da temperatura sobre os carboidratos totais. Segundo Celestino (2010), as hexoses, monossacarídeos com seis carbonos, como a glicose e a frutose são mais reativos que os dissacarídeos como a sacarose e a lactose.

\subsection{Carotenoides totais}

A desidratação altera os aspectos visuais da superfície do alimento, principalmente sua cor e refletância. A pupunha desidratada apresentou diferenças significativas nos teores de carotenóides totais para os três tratamentos, com $27,03,24,89$ e $20,98 \mu \mathrm{g} / \mathrm{g}$, respectivamente. Observa-se, que houve uma concentração maior de carotenóides em relação a pupunha in natura, no tratamento de secagem a $45^{\circ} \mathrm{C}$ e com o

Agropecuária Técnica, Areia-PB, v. 41, n. 3-4, p. 101-108, 2020

https://doi.org/10.25066/agrotec.v41i3-4.54435 
aumento de temperatura os teores, diminuíram. Esses resultados, estão próximo de $24,91 \mu \mathrm{g} / \mathrm{g}$ de carotenóides descrito para farinha de pupunha sem casca e muito abaixo de $74 \mu \mathrm{g} / \mathrm{g}$ para farinha de pupunha com casca (Kaefer te al., 2013). Aquino et al. (2009), citam que a secagem da polpa de pequi a $40^{\circ} \mathrm{C}$, preservou melhor as características nutricionais do fruto, devido à maior retenção de carotenóides. Segundo Celestino (2010), pigmentos como clorofila e carotenóides sofrem grandes modificações químicas causadas pelo processo de cocção. Entretanto, seus teores podem variar em decorrência de fatores como, estádio de maturação, condições edafoclimáticas do local de cultivo, grande número de carotenoides existente, suscetibilidade a isomerização e oxidação, dentre outros (Rodriguez-Amaya, 2004).

\section{Conclusões}

A temperatura mais eficiente na retirada de água livre da polpa de pupunha foi a $65^{\circ} \mathrm{C}$. Apesar dos lipídeos apresentarem-se mais concentrados a temperatura de $45^{\circ} \mathrm{C}$, foi a $65^{\circ} \mathrm{C}$ que houve maior concentração de proteínas e fibras. A polpa desidratada a 55 e $65^{\circ} \mathrm{C}$, podem ser consideradas como fonte de fibra alimentar segundo a legislação. O menor teor de cinzas, foi obtido no tratamento a temperatura de $45^{\circ} \mathrm{C}$. Os carboidratos e os carotenóides foram significativamente influenciados pelas maiores temperaturas de secagem. Portanto, dependendo da finalidade nutricional ou tecnológica para desidratar $o$ fruto de pupunha, as três temperaturas analisadas nesse estudo, devem ser utilizadas para atingir o objetivo ao qual a farinha de pupunha desidratada deve ser utilizada.

\section{Referências}

Andrade, J. S.; Pantoja, L.; Maeda, R. N. Melhoria do rendimento e do processo de obtenção da bebida alcoólica de pupunha (Bactris gasipaes Kunth). Campinas: Ciência e Tecnologia de Alimentos. v. 23, n. Supl, p. 3438 ,

2003.

https://www.scielo.br/pdf/cta/v23s0/19467.pdf

Aquino, L. P.; Ferrua, F. Q.; Borges, S. V.; Antoniassi, R.; Correa, J. L. G.; Cirillo, M. A. Influência da secagem do pequi (Caryocar brasiliense Camb.) na qualidade do óleo extraído. Ciência e Tecnologia dos Alimentos, v. 29, n. 2, p. 354-357, 2009. https://doi.org/10.1590/S0101-20612009000200018

Brasil. Ministério da Saúde. Agencia Nacional de Vigilância Sanitária. Resolução RDC n ${ }^{\circ} 263$, de 22 de setembro de 2005. Aprova o regulamento técnico para produtos de cereais, amidos, farinhas e farelos. Diário Oficial [da] República Federativa do Brasil, Brasília, DF, out. 2000. Disponível em: < http://www.anvisa.gov.br >. Acesso em: 24 julho 2019.

Brasil. Ministério da Saúde. Portaria n ${ }^{\circ}$ 27, de 13 de janeiro de 1998. Aprova o regulamento técnico referente à informação nutricional complementar. Diário Oficial da União. Poder Executivo, Brasília, DF, 16 de Janeiro de 1998. Disponível em: < http://bvsms.saude.gov.br/bvs/saudelegis/svs1/1998/prt00 27_13_01_1998.html > Acesso em: Agosto de 2020.

Carvalho, A. V.; Beckman, J. C.; Maciel, R. A.; Neto, J. T. F. Características físicas e químicas de frutos de pupunheira no estado do Pará. Revista Brasileira de Fruticultura, v. 35, n. 3, p. 763-768, 2013. https://ainfo.cnptia.embrapa.br/digital/bitstream/item/927 70/1/Artigo-Publicado-pupunha.PDF

Carvalho, A. V.; Vasconcelos, M. A. M. de.; Silva, P. A.; Ascheri, J. L. R. Produção de snacks de terceira geração por extrusão de misturas de farinhas de pupunha e mandioca. São Paulo: Brazil Journal Food Technology, v. $12, \quad$ n. 4, p. 277-284, 2009. https://doi.org/10.4260/BJFT2009800900022

Cecchi, H. M. Fundamentos teóricos e práticos em análise de alimentos. $2^{\mathrm{a}}$ ed. Rev. Campinas, SP: Editora da UNICAMP, 207p. 2003.

Celestino, S. M. C. Princípios de secagem de alimentos. Planaltina, DF: Embrapa Cerrados, 2010, 51p. (Documentos / Embrapa Cerrados, 276).

https://ainfo.cnptia.embrapa.br/digital/bitstream/item/777 65/1/doc-276.pdf

Clement, C. R. Amazonian Fruits: a neglected, threatened and potentially rich resource requires urgent attention. Diversity, v. 7, n. 1\&2, p. 56$59 \quad$ (USA), 1991. https://www.inpa.gov.br/cpca/charles/crc-cref.html

Ferreira, C. D.; Pena, Rosinelson, S. Comportamento higroscópico da farinha de 
pupunha (Bactris gasipaes). Campinas: Ciências e Tecnologia de Alimentos, v. 23, n. 2, p. 251-255, 2003. http://dx.doi.org/10.1590/S010120612003000200025

Ferreira, D F. SISVAR - Sistema de análises de variância para dados balanceados: programa de análises estatísticas e planejamento de experimentos. Versão 4.3. Lavras: UFLA, 2000.

Gallardo, V. M. S.; Sierra, C. E. M.. Condiciones de secado para la obtención de harina de chontaduro (Bactris gasipaes ). In: Congreso Internacional Sobre Biologia, Agronomia E Industrializaciión Del Pijuayo, 4., 1991, Iquitos. Anais... San José: Universidad de Costa Rica, $1993 . \quad$ p. 294-295. https://www.inpa.gov.br/pupunha/bibliografias/bib5agroin.html

Goia, C. H.; Andrade, J. S.; Aragão, C. G. Composição química da farinha de pupunha. Revista química industrial, v. 2, n. 2, p. 4854, 1993. https://repositorio.inpa.gov.br/handle/1/22922

Instituto Adolfo Lutz (IAL). Métodos físicoquímicos para análise de alimentos. 4.ed. 1.ed. digital. São Paulo: IMESP, 2008. 1020p. https://wp.ufpel.edu.br/nutricaobromatologia/files/2013/0 7/NormasADOLFOLUTZ.pdf

Kaefer, S.; Fogaça, A. O.; Storck, C. R.; Kirsten, V. R. Bolo com farinha de pupunha. Brazilian Journal Food Nutrition., v. 24, n. 3, p. 347352, 2013. https://docplayer.com.br/67976230-Bolocom-farinha-de-pupunha-bactris-gasipaes-analise-dacomposicao-centesimal-e-sensorial.html

Kumar, C.; Karim, M. A.; Joardder, M. U. H. Intermittent drying of food products: A critical review. Journal of Food Engineering, v. 121, p. 48-57, 2014. https://doi.org/10.1016/j.jfoodeng.2013.08.014

Lima, D. G. de.; Silva, R. F. da.; Furtado, M. T. Composição química e aspectos microbiológicos de pães enriquecidos com polpa integral de pupunha desidratada. Revista GEINTEC，v. 10, n. 1, p. 5352-5366, 2020. https://doi.org/10.7198/geintec.v10i1.1374

Martínez-Girón, J.; Rodrígues-Rodrígues; X., Pinzón-Zárate, L. X., \& Ordónez-Santos, L. E. Caracterização físico-química de farinha de resíduos do fruto de pupunha (Bactris gasipaes Kunth, Arecaceae) obtida por secagem convectiva. Ciência \& Tecnologia Agropecuária., v. 18, n. 3, p. 599-613, 2017. http://dx.doi.org/10.21930/rcta.vol18_num3_art:747
Oliveira, A. M. M. M.; Marinho, H. A. Development of panettone made of peach palm flour (Bactris gasipaes Kunth). Brazilian Journal of Food and Nutrition, v. 21, n. 4, p. 595-605, 2010.

Oliveira, A. N.; Oliveira, L. A. de.; Andrade, J. S.; Chagas-Júnior, A. F. Produção de amilase por rizóbios, usando farinha de pupunha como substrato. Ciência e Tecnologia de Alimentos, v. 27, n. 1, p. 61-66, 2007. https://doi.org/10.1590/S0101-20612007000100011

Parante, G. D. L.; Pinto, S. M.; Santos, I. dos. Obtenção da polpa desidratada do pequi (Caryocar brasiliense) destinada a comercialização na região do cariri. BioFar. Revista de Biologia e Farmácia, v. 10, n. 1, p. $48-54$ 2014. http://revista.uepb.edu.br/index.php/biofarm/article/view/ 2393

Park, K. J; Yado, M. K. M; Brod, F. P. R. Estudo de secagem de pêra bartlett (Pyrus sp.) em fatias. Ciência e Tecnologia de Alimentos, v. 21, n. 3, p. 288-292, 2001. https://www.scielo.br/pdf/cta/v21n3/8545.pdf

Reck, I. M.; Miranda, N. L. de. Composição química e qualidade microbiológica de formulações de biscoitos com farinha de polpa de pupunha. Revista Uningá, Maringá, v. 27, n. 1 , p. 15-18, 2016. http://revista.uninga.br/index.php/uningareviews/article/vi ew/1816/1421

Rodriguez-Amaya, D. B. Avanços na pesquisa de carotenóides em alimentos: contribuições de um laboratório brasileiro. Revista do Instituto Adolfo Lutz, v. 63, n. 2, p. 129-138, 2004. https://pesquisa.bvsalud.org/portal/resource/pt/lil-404791

Rojas-Garbanzo, C.; Perez, A. M; Vaillant, F.; Pineda-Castro, M. L. Physicochemical and antioxidant composition of fresh peach palm (Bactris gasipaes Kunth) fruits in Costa Rica. Brazilian Journal of Food Technology, v. 19, e2015097, 2016. http://dx.doi.org/10.1590/1981-6723.9715

Santos, B. W. C. dos.; Ferreira, F. M.; Souza, V. F. de.; Clement, C. R.; Rocha, R. B. Análise discriminante das características físicas e químicas de frutos de pupunha (Bactris gasipaes Kunth) do alto Rio Madeira, Rondônia, Brasil. Científica, v. 45, n. 2, p. 154$161, \quad 2017 . \quad$ http://dx.doi.org/10.15361/1984$5529.2017 \mathrm{v} 45 \mathrm{n} 2 \mathrm{p} 154-161$ 
Silva, L. M. M.; Souza, F. C. de.; Deise, S. C.; Nunes, J. S.; Almeida, F. A. C. Avaliação das Características Físicas e Físico-Químicas da Pupunha. Revista Verde, v. 8, n. 3, p. 5-8, 2013.

https://www.gvaa.com.br/revista/index.php/RVADS/artic le/view/2018/1734
Souza, J. L. F.; Oliveira, D. E. C.; Placido, G. R.; Egea, M. B.; Caliari, M.; Silva, M. A. P. da. Thermodynamic and nutritional properties and drying kinetics of pequi (Caryocar brasiliense Cambess) mesocarp. Revista Brasileira de Engenharia Agrícola e Ambiental, v. 23, n. 9, p. 655-661, 2019. http://dx.doi.org/10.1590/18071929/agriambi.v23n9p655-661 . 